\title{
Indometacina en el tratamiento del ductus arterioso persistente en recién nacidos prematuros
}

\author{
Patricio Ventura-Juncá del T.' ; Alvaro González M.' ; \\ Noemí Arrizaga G. ${ }^{1}$
}

\section{Indomethacin versus maintenance treatment in newborn infants with patent ductus arteriosus}

\begin{abstract}
The effectiveness of indomethacin versus usual medical treatment with fluid restriction to $75 \%$ of the volume given in the previous davs. with or without frusemide (UMT), for the closure of hemodynamically signiflcant patent ductus arteriosus (HSPCA) was retrospectively studied in 87 premature infants under $2000 \mathrm{~g}$ admitted to our nursery from year 1978 throughout 1988. Among 16 babies, treated from the begining with indomethacin and UMT, HSPDA was closed 72 h later in 14 (87.5\%), versus only 14 closures in 71 babies (19.7\%) treated $\mathrm{by}$ UMT alone ip $<0.01 \%$. Of the 57 babies whose HSPDA failed to close with UMT alone, 23 were given indomethacin and successful closure was seen in 16 (69.6\%), while among 34 babies remaining on UMT alone, closure resulted in only $7(20.6 \%)(p<0.01)$. Intravenous route for administration and early use of indomethacin (before age 7 days) proved to have significantly higher success rates than oral route and use of the drug after age 7 days.

(Kev words: patent ductus arteriosus, indomethacin, newborns.)
\end{abstract}

La persistencia del ductus arterjoso (DAP) está asociada con importante morbilidad en prematuros, especialmente aquellos con afecciones o trastornos respiratorios ${ }^{1-3}$. $\mathrm{Si}$ el cortocircuito de izquierda a derecha por el ductus es importante, produce exceso de flujo pulmonar, eventualmente falla ventricular izquierda y ambos en conjunto afectan desfavorablemente la resistencia vascular y distensibilidad del pulmón ${ }^{4,5}$. Esto es especjalmente notorio en los rihos de muy bajo peso, como lo confirman estudios con surfactante exógeno, pues la mejoría en la función pulmonar que éstos producen está limitada por la intensidad del flujo de jzquierda a derecha a través del ductus ${ }^{6,7}$. En algunos recién nacjdos prematuros, especialmente aquellos sin afecciones respiratorias, el DAP se cierra espontáneamente ${ }^{1,8,9}$. La incidencia de DAP aumenta en proporción inversa a la edad gestacional. En nuestra unjdad, en niños $<1000 \mathrm{~g}$ que sobreviven a las primeras 24 horas es de $47,2 \%$ y en cerca de $90 \%$ de ellos se asocia con enfermedades respiratorias ${ }^{10}$. En muchos de estos niños el DAP juega un rol decisivo en la deficiente fun-

1. Departamento de Pediatría, Unidad de Neonatología, Hospital Clínico Universidad Católica de Chile. ción cardiovascular y respiratoria y en la consiguiente demora en el retiro de la ventilación mecánica. Además el DAP ha sido asociado a otros problemas neonatales como hemorragia intracraneana ${ }^{14}$, enterocolotis necrótica, displasia broncopulmonar y dismjnución de la filtración glomerular $^{11-13}$. Por estas razones se han busca. do diversos tratamientos para lograr el cierre del DAP en prematuros. En el cierre del ductus in. fluyen diversos factores, entre ellos, la presión parcial de oxígeno ${ }^{19}$, las prostaglandinas y el aporte hidrico ${ }^{19-23}$. Mientras menor es la edad gestacional, menor es la sensibilidad del ductus a contraerse frente al oxígeno, y mayor es su sensibilidad a la acción de las prostaglandinas que lo mantienen permeable ${ }^{20,24,26}$.

Existe suficiente evidencia sobre la efectividad de la administración de indometacina, un inhibidor de la síntesis de prostaglandinas, en el cierre farmacológico del DAP en prematu$\operatorname{ros}^{27-30}$, razón por la cual, al tratamiento médico usual -basado en la restricción de líquidos y uso de diuréticos (TMU) - y al cjerre quirúrgico se ha agregado el empleo de indometacina. El objetivo de este trabajo fue describir la experiencja acumulada en una unidad de neonatología, entre los affos 1978 y 1988 , en el uso de indometacina, comparándola con los resulta- 
dos del tratamiento usual, sin esta droga, para cerrar el DAP. Además se intentará comparar los resultados obtenidos por distintas vías de administración (oral y endovenosa) y con distintas dosis del medicamento.

\section{Material y Método}

En nuestra unidad se usó por primera vez indometacina en 1978 en base a las primeras publicaciones sobre el tema ${ }^{27}$ y los protocolos aplicados fueron cambiando según los resultados que se publicaban, lo que explica los diferentes esquemas de dosis y vías de administración empleados. Como no se contaba en ese entonces con facilidades para el cierre quirúrgico del ductus, los medios farmacológicos fueron la única opción para los niños en que fracasaba el tratamiento nédico usual.

Se revisaron las historias clínicas de todos los RN $<2000 \mathrm{~g}$, hospitalizados en la unidad de neonatología del Hospital Clínico de la Universida đatólica de Chile entre el $1^{\circ}$ de encro de 1978 y el 31 de diciembre de 1988, que cumplían los criterios -especificados en otro estudio previo sobre incidencia ${ }^{10}$ - de ductus arterioso permeable hemodinámicamente significativo (DAPHS).

Los esquemas terapéuticos empleados fueron los sigujentes: en 71 RN usó tratamiento médico usual, que consistió en restricción de líquidos (aporte $\leqslant 75 \%$ del volumen aportado en los días precedentes), asociado o no al uso de diurêticos (furosenjia); en 23 niños se usó indometacina después de haber fracasado el TMU y en 16 en forma simultánea con éste, dentro de las primeras 48 horas de identificado el DAP. En 14 pacientes la indometacina se usó por vía oral y en 25 por vía endovenosa. Las dosis por vía oral variaron entre 0,1 y $1 \mathrm{mg} \cdot \mathrm{kg}$ y por vía endovenosa entre 0,1 y $0,3 \mathrm{mg} \cdot \mathbf{k g}$ dosis. La mayoría de los pacientes recibió entre 2 y 3 dosis, separadas por 8 a 12 horas. En 34 recién nacidos no se pudo usar indometacina porque estaba contraindicado o no se disponía de elta. Se consideraron contraindicaciones: recuentos de plaquetas $<60000 \times \mathrm{mm}^{3}$ de sangre; evidencia de diatesis hemorrágica; hemorragia intracraneana reciente; enterocolitis necrótica en evolución; signos de disfunción renal: creatininemia $>\mathbf{1 , 8 \%} \mathrm{mg} / \mathrm{dl}$, N ureico sanguineo $>30 \mathrm{mg} / \mathrm{dl}$ u oliguria $(<\mathrm{I} \mathrm{ml} \cdot \mathrm{kg} \cdot \mathrm{h}$ en las horas previas). La hiperbjlirrubinemia se conskiteró contraindicación en los primeros años de la serie. El tratamiento se calificó de buen éxito cuando el soplo desapareció dentro de las prìmeras 72 h de iniciado éste, manteniéndose ausente por al menos $48 \mathrm{~h}$. Se consideró reapertura del ductus la reaparición del soplo 48 h o más después de su desaparición.

Para el análisis estadístico de los resultados se usó la prueba de $\chi^{2}$ cuando procedía. Se consideró estadisticamente significativo un valor $\mathrm{p}<0,05$.

\section{Resultados}

De 1 los 90 pacientes que cumplieron con los critenos de DAPHS, 87 recibjeron tratamiento y 3 no alcanzaron a tratarse porque fallecieron precozmente. En 71 de los niffos tratados se usó inicialmente sólo TMU, obteniéndose buen éxito en $14(19,7 \%)$. En 16 pacientes se usó inicialmente indometacina asociada con TMU, lográndose buen éxjto en $14(87,5 \%)$. La diferencia de efectividad entre ambos tratamientos fue estadisticamente significativa ( $\mathrm{p}<0,001$ ). De los 57 casos en que fracasó el TMU inicial, se usó indometacina en 23 , con buen resultado en $16(69,6 \%)$. En los 34 restantes se insistió en el TMU, mencionandose cierre del ductus sólo en $7(20,6 \%)$. La diferencia es también estadisticamente significativa $(\mathrm{p}<0,001)$.

En 14 pacientes se usó indometacina por vía oral y el ductus se cerró en $7(50 \%)$ de ellos. De los 25 casos en que se usó por vía endovenosa se logró buen éxito en 23 (92\%). La diferencia es nuevamente significativa $(\mathrm{p}<0,01)$. La distribución de los pacientes por peso de nacimiento fue semejante para ambas vías, pero el momento de administración fue más tardio cuando se empleó la oral (11 vs. 7 días).

En todos los 18 recién nacidos en que se usó la indometacina precozmente (dentro de la primera semana de vida), se logró el cierre del ductus, en contraste con sólo $9(42,9 \%)$ buenos resultados entre los 21 casos en que el medicamento se usó más tarde $(p<0,01)$. Sin embargo, hay que destacar que el ductus se cerró en 3 de 4 pacientes en que la indometacina se usó después de los 20 días de vida.

De los 30 niños en que el DAPHS se cerró en coincidencia con el uso de indometacina, en $11(36,7 \%)$ se registró reapertura del conducto, 5 de éstos con cortocircuito hemodinámicamente significativo. La reapertura fue más frecuente e importante en los niños que pesaban menos de $1000 \mathrm{~g}$ al nacer, entre quienes -de 11 tratados- el ductus se reabrió en 6 , siendo en 4 hemodinámicamente significativo, en contraste con 5 reaperturas entre 19 niños de 1000 a $2000 \mathrm{~g}$, sólo en un caso hemodinámicamente significativa. En 4 pacientes, todos menores de $1000 \mathrm{~g}$ de peso al nacer, se usó indometacina por segunda vez $y$ en dos por tercera vez. Uno de ellos falleció con DAPHS y otro sobrevivió con soplo persistente no significativo. Los otros dos casos sobrevivieron, cerrándose el ductus durante TMU o en forma espontánea.

En 18 de 39 casos $(46,2 \%)$ se registró disminución transitoria de la djuresis, con oliguria en $4(10,3 \%)$. En 7 pacientes se registró ascenso 
del $\mathrm{N}$ ureico sanguíneo sobre $25 \mathrm{mg} / \mathrm{dl}(17,9 \%)$, que en la mayorja fue moderado y en sólo dos niños superior a $50 \mathrm{mg} / \mathrm{dl}$. En cuatro recién nacidos la reacción de guayaco para sangramientos ocultos dio resultados positivos después de la administración de indometacina; en todos fue un fenómeno transitorio. En ringuno hubo evidencias de sangramiento externo en coincidencia con el medicamento.

\section{Comentario}

Desde que se comunicaron en 1976 las experiencias con inhibidores de las prostaglandinas para cerrar el DAP en niños prematuros ${ }^{21,28}$, un número importante de estudios posteriores confirmaron su efectividad ${ }^{23,30}$, de la misma manera que esta experiencia. El tratamiento con indometacina simultáneamente con TMU, desde el momento en que se diagnostica DAPHS, tiene mayor porcentaje de buenos éxitos que cuando la droga se usa después de fracasado el TMU. Este último tiene los riesgos adicionales de que, durante su curso, aparezcan complicaciones que contraindiquen el empleo de indome. tacina $y$ aumenten los efectos deletéreos del DAP en la mecánica pulmonar. La indometacina ha sido administrada con buenos resultados por vía oral y endovenosa ${ }^{30}$. Algunos han serialado, para la vía oral, dificultades derivadas de la relativa falta de solubilidad, técnica de administración y absorción del medicamento ${ }^{31}$. La famacoqujnética de la indometacina por vía endovenosa es bien conocida y permite establecer una buena relación entre dosis y niveles plasmáticos esperados a distintas edades gestacionales. En nuestra experiencia, si bien los grupos de pacientes no son estrictamente comparables, son mejores los resultados por vía endovenosa, en concordancia con las ventajas recién descritas y la mayoría de las recomendaciones ${ }^{32}$. El resultado es significativamente mejor cuando la indo. metacina se usa en la primera semana de vida. Sin embargo, a pesar que después de la primera semana el porcentaje de éxito es menor, éste es superior al de otras experiencias y, aun cuando algunos han expresado que el medicamento es inefectivo en niños prematuros con más de 2 semanas de vida, nosotros hemos tenido buen éxito más tarde en varios de estos pacientes, hecho que también ha sido comunicado por otros recientement $\mathrm{e}^{33}$. La reapertura del DAP después del tratamiento exitoso con indometacina fue más frecuente y de mayor significación hemodinámica mientras menor el peso de nacimiento del nifto, lo que también concuerda con otras experiencias ${ }^{29}$, posiblemente relacionada con mayor sensibilidad del ductus al efecto vasodilatador de las prostaglandinas y más incidencia de trastornos respiratorios graves en los nifnos más prematuros. La frecuencia de reapertura encon. trada en este estudio, especialmente en los nj. fios mayores de $1000 \mathrm{~g}$, es mayor que la comunicada por otros 29 . Es posible que esto se deba a que en algunos casos no se emplearon las tres dosis de indometacina recomendadas actualmente.

Se han descrito varios efectos adversos de la indometacina, entre los que destacan grados variables de disfunción renal ${ }^{18,34}$ y sangramiento por alteración de la agregación plaquetaria. En nuestra casuística se observó alza discreta y transitoria del BUN y creatinina, acompanados de moderada disminución de la diuresis, pero pocos ninos presentaron oliguria $y$ alza del N urejco sobre $50 \mathrm{mg} / \mathrm{dl}$, en todos transitorias. La adecuada vigilancia del balance hidrico y la función renal contribuyeron a la poca relevancia de este efecto colateral. En nuestros pacientes no encontramos evidencias de sangramiento macroscópico. El hecho de asegurarse de un número adecuado de plaquetas antes de indicar la jndometacina y la contraindicación de su uso en nif́os que presentaban signos hemorragiparos, probablemente contribuyó a minimizar este riesgo.

En conclusión, esta experiencia confirma los buenos resultados del tratamiento del DAPHS con indometacina. De acuerdo con la experiencia acumulada ${ }^{35}$, las recomendaciones de dosis e indicaciones para el empleo de indometacina son actualmente las siguientes: todos los prematuros cuyo peso al nacer sea $<1000 \mathrm{~g}$, en el momento en que aparece el soplo con características de DAP. La experiencia demuestra que casi todos los ductus arteriosos persistentes serán hemodinámicamente significativos en algún momento. Todos los prematuros con peso de nacimiento $<1500 \mathrm{~g}$, que requieren ventilación mecánica en los primeros días de vida, en el momento en que aparece un soplo con características de DAP. Todos los prematuros en que aparezca un soplo con características de DAP en el momento en que éste produzca sig. nos de trastomo hemodinámico. Todos los 
casos de DAP silente diagnosticado por ecocardiografía, en que se estén produciendo trastornos hemodinámicos. Las dosis de indometacina recomendadas para prematuros de $<48$ horas de vida son de $0,2 \mathrm{mg} \cdot \mathrm{kg}$ la primera, seguida por dos dosis de $0,1 \mathrm{mg} \cdot \mathrm{kg}$ cada una, suministradas cada $12 \mathrm{~h}$. Para prematuros de 2 a 7 dias de vida se recomjenda usar $0,2 \mathrm{mg} \cdot \mathbf{k g}$ en la primera dosis, seguida de otras dos iguales a la anterior cada $12 \mathrm{~h}$. En prematuros de $>7$ días de vida, la primera dosis será de $0,2 \mathrm{mg} \cdot \mathbf{k g}$, seguida de otras dos de $0,25 \mathrm{mg} \cdot \mathrm{kg}$ cada $12 \mathrm{~h}$.

\section{Resumen}

Se estudió en forma retrospectiva la eficacia de la indometacina en el cierre del ductus arterioso persistente hemodinámicamente significativo, comparándola con la del tratamiento médico usual (TMU), en 87 prematuros menores de $2000 \mathrm{~g}$ atendjdos en una unidad de neonatología en un centro clínico universitario, entre los años 1978 y 1988. En 16 nifios en que se usó indometacina desde el inicio, asociada con TMU, se habia logrado, $72 \mathrm{~h}$ después, buen éxj. to en $14(87,5 \%)$, resultado signifjcativamente mejor al cierre logrado en 14 de 71 niños $(19,7 \%)$ que recibieron sólo TMU $(\mathrm{p}<0,01)$. De los 57 nif̃os en que el TMU había fracasado a las 72 $h, 23$ recibieron indometacina y se obtuvo el cierre del ductus en $16(69,6 \%)$, mientras entre los 34 restantes que continuaron sólo con TMU este efecto sólo se registró en $7(20,6 \%)$, lo que es significativamente menor $(p<0,01)$. La via intravenosa $y$ el uso precoz de indometacina (antes de 7 días) demostró tener un éxito significativamente mejor que la vía oral y el uso tardio de esta droga.

(Palabras clave: đuctus arterioso permeable, indometacina, recién nacidos, prematuros.)

\section{Referencias}

1. Kitteman JA, Edmunds LH, Gregory GA, Heymann MA, Tooley WH, Rudolph AM: Patent ductus arteriosus in low-birthweight infants: Incidence, relation to pulmonary disease and management. N Engl J Med 1972; 287: 473-477.

2. Krovetz $L I$, and Rowe $R D$ : Patent ductus, prematurity and pulmonary disease; Editorial, $N$ Engl J Med 1972; 287:513.

3. Thibeault $D W$, Emmanouledes $G C$, Nelson $R J$, Lochmon RS, Rosengart RM, on W: Patent ductus arteriosus complicating the respiratory distress syndrome in preterm infants. J Pediatr 1976; 86: $120-126$.

4. Alverson $D C$, Eldridge $M W$, Johnson JD: Effects of patent ductus arteriosus on left ventticular output in premature infants. J Pediatr 1983; 102: 754-757.

5. Noulty $C M$, Hom $S$, Conry $J_{1}$ Avery $G B$ : Improved lung compliance after ligation of patent ductus arteriosus in hyaline membrane disease. J Pediatr 1978;93: 682.

6. Fujiwara $T$ Chida $S$, Watabe $Y$, Maeta $H$, Morito $T$ : Abet. Artificial surfactant therapy in hyalinemembrane disease. Lancet $8159: 1980: 55-59$.

7. Merrit TA, Hollman $M$, Sprogg $R$ et al.: Exogenous Surfactant Treatments for Neonatal Respiratory Distress Syndrome. Drugs 1989; 38: 591-611.

8. Auld PAM: Delaged closure of the ductus arteriosus. J Pediatr $1966 ; 69 ; 61-66$.

9. Gentile R. Stevenson GM, Dooley T, Franklin D, Kawabori f. Peariman A: Pulsed Doppler echocardiographic determination of time of ductal closure in normal newborn infants. J Pediatr 1981; 98: 443-448.

10. González $A$. Ventura Juncá $P$ : Incidencia del ductus arterioso persistente clínicamente aparente en prematuros de menos de $2000 \mathrm{~g}$. Rev Chil Pediatr (en prensa).

11. Siassi B, Emmanouildes $G C$, Clevelond RJ, and Hiros F: Patent ductus arteriosus complicating prolonged assisted ventilation in respiratory distress syndrome. J Pediatr 1969; 74: 11-19.

12. Dudell GG, Gersony WM: Patent ductus arteriosus in neonates with severe respiratory disease. J Pediatr 1984; 104: $915-920$.

13. Lipman B, Serwer GA, Brazy JE: Abnormal cerebral hemodynamics in preterm infants with patent ductus arteriosus. J Pediatr 1982;69: 778.

14. Martin CG, Snider AR, Katz SM etal, Abrormal cerebral blood flow patterns in preterm infants with a large patent ductus arteriosus. I Pediatr 1982; 101: 587 .

15. Ryder $R W$, Shelton $D$. Guinan $M E$ : Necrotizins enterocolitis: A prospective multicenter investigation. Am J Epidern iol 1980; 112: 113.

16. Brown ER: Increased risk of bronchopulmonary displasia in infants with patent ductus arteriosus. J Pediatr 1979; 95 : 865-866.

17. Bancalari E, Sosenko I: Pathogenesis and Prevention of Neonatal Chronic Lung Disease: Recent Developments. Pediatric Pulmonology 1990; 8 : 109.116.

18. Seyberth $J W$, Rascher $W$, Hackenthal $R$, wille $L$ Effect of prolonged indomethacin therapy on renal function and selected vasoactive hormones in very-low-birth-weight infants with symptomatic patent ductus arteriosus. J Pediatr 1983; 103: 979-984.

19. Kowalcik $V$ : The response of the isolated ductus arteriosus to oxygen and anoxia. J Physiol (Lond) 1963;169: 185 .

20. Clyman RI. Heymann MA: Pharmacology of the ductus arteriosus. Pediatr Clin North Am 1981; 28: 77-93. 
21. Cyman $R I$, Heymann AM: Ductus arteriosus responses to prostaglandin. $\mathrm{El}$ at high and low oxygen concentrations. Prosaglandins, 1977; 13: 219.

22. Coceani $F$, olley PM: The response of the ductus arteriosus to prostaglandins. Can J Physiol Pharmacol 1973:51:220-225.

23. Bell EF, Warburton D, Stones Treet BS, Oh W: Effect of fluid administration on the development of symptomatic patent ductus arteriosus and congestive heart fajure in premature infants. N Eng] J Med 1980; 302: 598-604.

24. Noel $S$, Cassin $S$ : Maluration of contractile response of the ductus arteriosus to oxygen and drugs. Am J Physiol 1976; $231: 240$.

25. Cyman RI. Mauray $F$, Wong $L$ ef al: The developmental response of the ductus arteriosus to oxygen. Biol Neonate 1978; 34: 177.

26. Cyman RI, Mauray F. Roman L, Heymanit MA, Poyne B: Factors determining the loss of ductus arteriosus responsiveness to prostaglandin $E$. Circulation $1983 ; 68: 433$.

27. Friedman WF, Mirschklon MJ, Printz MP, Pillick PT, Kirkparrick SE. Pharmacologic closure of patent ductus arteriosus in the premature infant. N Engl I Med 1976; 295 : 526-529.

28. Heymann MA, Rudolph AM. Silverman NH: Closure of the ductus arteriosus in premature infants by inhibition of prostaglandin synthesis. N Engl J Med 1976; 295: 530-533.

29. Gersony WM, Pecknam GJ, Ellison RC, Miettinen $O S$, Nadas $A S$ : Effects of indomethacin in premature infants with patent ductus arteriosus. Results of a national collaborative study, J Pe. diatr 1983; 102 : 895-906.

30. Barst RJ, Gersony WM: The Pharmacological treatment of Patent Ductus Arteriosus. A review of the eyjdence. Drugs $1989 ; 38: 249-266$.

31. Bhat $R$, Vidyasar $D$. Vodapalli $M$ et al.: Disposition of indomethacin in preterm infants. J Pediatr $1979 ; 95: 313$.

32. Taljf $A A$, Carr $I$, Yeht $T$ y Fet al.: Pharmacokinetics of intravenous administered indomethacin in premature infants. J Pediatr 1980; 97: 995. 1000 .

33. Kresch MJ, Moya FR, Ascuitto RJ, Ross-Ascuitto NT, Heusser F: Late closure of the ductus ar. teriosus using indomethacin in the preterm infant. Clin Pediatr 1988; 27: 140-143.

34. Winter $A$, Tapia $J L$, Ventura-Juncá $P$ et al.: Falla renal aguda por sobredosis de Indometacina en un tecièn nacido prematuro con un ductus arterioso persistente. Rev Chil Pediatr 1986; 57: 444-446.

35. Clyman RI, CampbelI D: Indomethacin therapy for patent ductus arteriosus: When is prophylaxis not prophylactic? J Pedjatr 1987; 718-722. 